\title{
The miR-200 family regulates the epithelial-mesenchymal transition induced by EGF/EGFR in anaplastic thyroid cancer cells
}

\author{
ZHE ZHANG $^{1}$, ZE-BING LIU ${ }^{2}$, WEI-MIN REN ${ }^{2}$, XUAN-GUANG YE ${ }^{2}$ and YOU-YUAN ZHANG ${ }^{2}$ \\ ${ }^{1}$ Department of Integrated Therapy, Fudan University Shanghai Cancer Center, and Department of Oncology, \\ Shanghai Medical College, Fudan University, Shanghai 200032; ${ }^{2}$ Department of Pathology, \\ Jinshan Hospital, Fudan University, Shanghai 200540, P.R. China
}

Received April 8, 2012; Accepted June 15, 2012

DOI: $10.3892 /$ ijmm.2012.1059

\begin{abstract}
The miR-200 family was recently identified as a suppressor of epithelial-mesenchymal transition (EMT). The loss or gain of miR-200 family members is associated with cancer invasion. The epidermal growth factor receptor (EGFR) is overexpressed in the majority of anaplastic thyroid cancers (ATCs). The activation of EGFR by its ligand, epidermal growth factor (EGF), activates a signaling cascade that results in the enhanced migration and invasiveness of thyroid cancer cells. However, little is known about the potential interrelationships between EGF/EGFR, miR-200s and the induction of EMT or mesenchymal-epithelial transition (MET) processes. This study aimed to investigate the regulatory role of miR200s in EMT modulation by EGF/EGFR. Using transfection, real-time reverse transcription PCR and western blot analysis, we found that the EGF treatment of Nthy-ori 3-1 thyroid follicular cells resulted in the downregulation of E-cadherin and the upregulation of vimentin. By contrast, EGFR silencing in SW1736 human thyroid carcinoma cells led to the upregulation of E-cadherin and the downregulation of vimentin. In addition, EGF signaling correlated with the reduced expression of miR-200s and the re-expression of miR-200s abrogated the effects of EGF treatment and restored an epithelial phenotype to EGF-induced Nthy-ori 3-1 cells. Conversely, the silencing of miR-200s in SW1736 cells overcame siEGFR-induced changes in gene expression and phenotype. In addition, we demonstrate that miR-200s play a key role in in vitro EGF/EGFR-mediated thyroid cell invasion and in EMT in vivo. We, therefore, provide a mechanistic link between the miR-200 family and
\end{abstract}

Correspondence to: Professor You-Yuan Zhang, Department of Pathology, Jinshan Hospital, Fudan University, No. 1508 Long Hang Road, Jinshan District, Shanghai 200540, P.R. China

E-mail: sszyys@yahoo.cn

Key words: miR-200, epithelial-mesenchymal transition, epidermal growth factor, epidermal growth factor receptor, anaplastic thyroid cancer cell
EGF/EGFR, which suggests that miR-200 upregulation may serve as a novel therapeutic strategy for highly invasive thyroid cancers.

\section{Introduction}

Anaplastic thyroid cancer (ATC) is one of the most aggressive types of malignant tumor, characterized by invasion into surrounding tissues and metastasis that contribute to a poor prognosis for patients with this disease (1-3). The acquisition of invasive and migratory properties is associated with epithelialmesenchymal transition (EMT) and is a prerequisite for cancer invasion into surrounding tissues, the first stage of metastatic disease (4-6).

The epidermal growth factor receptor (EGFR), a transmembrane cell-surface glycoprotein with intrinsic tyrosine kinase activity, is overexpressed in most ATCs (7). EGFR activation by its ligand, the epidermal growth factor (EGF), initiates a signaling cascade that results in changes in gene expression. EGF is the prototype of a large family of structurally related peptides that possess an EGF-like domain, consisting of 6 cysteine residues capable of forming 3 intramolecular loops stabilized by disulfide bonds. EGF is synthesized by the thyroid gland and can induce thyroid cell proliferation in a number of species. In addition, EGF enhances the migration and invasiveness of thyroid cancer (3,8-11).

The Rho/Rho kinase pathway may be important for cancer invasion, growth and metastasis. In vitro studies and animal experiments have suggested that the inhibition of the Rho/Rho kinase pathway inhibits tumor invasion and metastasis (12-16). Zhong et al (3) demonstrated that lovastatin suppresses the invasive capability of ATC cells by inhibiting Rho geranylgeranylation and RhoA/ROCK signaling. Rho/ ROCK signaling is required for EGF-induced invasion of ARO human ATC cells.

The expression of miR-200s is associated with tumor invasion and regulates EMT in cancer cells (17-20). However, nothing is known about interrelationships between EGF/ EGFR, miR-200s and the EMT or mesenchymal-epithelial transition (MET) processes. In the current study, we first show that the activation of EGF/EGFR signaling by EGF treat- 
ment of Nthy-ori 3-1 thyroid follicular cells downregulates E-cadherin with a concomitant upregulation of vimentin. Conversely, the downregulation of EGF/EGFR signaling by EGFR silencing in SW1736 cells results in E-cadherin upregulation and vimentin downregulation. Secondly, EGF treatment inversely correlates with the expression of miR-200 family members. Thirdly, re-expression of miR-200s in EGF-induced Nthy-ori 3-1 cells restores an epithelial phenotype, whereas the silencing of miR-200s in SW1736 cells reverses siEGFRmediated changes. Finally, miR-200s are shown to play a key role in EGF/EGFR-mediated thyroid cell invasion in vitro and EMT in vivo, suggesting that EGF/EGFR signaling regulates the aggressiveness of SW1736 cells by modulating miR-200 expression.

\section{Materials and methods}

Cell lines and reagents. The Nthy-ori 3-1 normal thyroid follicular epithelial cell line and the SW1736 human and ARO human ATC cell lines were obtained from the State Key Laboratory of Molecular Oncology, Chinese Academy of Medical Sciences (Beijing, China). All cells were cultured in RPMI-1640 medium supplemented with 5\% FBS, $100 \mathrm{U} / \mathrm{ml}$ penicillin and $100 \mathrm{mg} / \mathrm{ml}$ streptomycin in a humidified atmosphere with $5 \% \mathrm{CO}_{2}$ at $37^{\circ} \mathrm{C}$. All cell lines were tested and authenticated by short tandem repeat profiling using the PowerPlex 16 System (Promega). Antibodies were obtained from the following suppliers: vimentin (Abcam); E-cadherin, RhoA and $\beta$-actin (Sigma).

Real-time reverse transcription PCR (RT-PCR). Total RNA was isolated using TRIzol reagent (Invitrogen) according to the manufacturer's instructions. Real-time PCR was used to quantify mRNA expression. Primer sequences for E-cadherin, vimentin and glyceraldehyde-3-phosphate dehydrogenase (GAPDH) were as described previously (21), and specific gene expression was normalized to GAPDH. For miRNA analysis, total RNA was isolated using the mirVana miRNA isolation kit (Ambion) and the levels of miRNAs were determined using miRNA-specific TaqMan MGB probes from the TaqMan MicroRNA assay kit (Applied Biosystems). miRNA expression was normalized to RNU6B (Sigma).

Transfection experiments. Thyroid cells were seeded into 12-well plates and subjected to transfection with specific siRNAs targeting EGFR, pre-miR-200s (miR-200a, miR-200b and miR-200c), anti-miR-200s (Sigma), or scrambled controls by the liposome method, as described previously (8).

Western blot analysis. Western blot analyses were performed as previously described (9). Briefly, proteins were electrophoresed on $12 \%$ polyacrylamide gels and transferred to Hybond-P polyvinylidene difluoride (PVDF) membranes (Amersham). Western blot analysis was carried out with specific primary antibodies diluted in $1 \%$ bovine serum albumin (BSA) in TBST, followed by peroxidase-conjugated secondary antibody. Target proteins were observed using the enhanced chemiluminescence (ECL) detection system (GE Healthcare) and autoradiography on Fuji super RX film (Fuji, Tokyo, Japan), with 1-2 min exposure.
Cell invasion assay. Cell invasion through a reconstituted Matrigel basement membrane was assayed as previously described (22). Briefly, polycarbonate membranes $(8.0 \mathrm{~mm}$ pore size) in the base of the upper compartment of Transwell culture chambers were coated with $10 \%$ Matrigel (50 $\mu \mathrm{l} /$ insert), and the lower compartment was filled with $600 \mu \mathrm{l}$ serum-free medium containing $0.1 \%$ BSA. Cells were suspended in serum-free medium and seeded into the Transwell inserts. After $24 \mathrm{~h}$, cells that had invaded the Matrigel membrane were stained with $4 \mathrm{mg} / \mathrm{ml}$ calcein AM (Invitrogen) in PBS at $37^{\circ} \mathrm{C}$ for $1 \mathrm{~h}$ and then photographed under a fluorescent microscope. Invading cells were then trypsinized to remove them from the inserts. The images were recorded and analyzed using a Zeiss confocal photomicroscope LSM510 (Zeiss).

Immunohistochemistry. Immunohistochemistry analysis was performed as previously described (23). Paraffin-embedded tissues were cut into $4 \mu \mathrm{m}$ thick sections. Endogenous peroxidase activity was then blocked with $3 \%$ hydrogen peroxide, and then endogenous biotin was blocked with $0.01 \%$ avidin. Following blocking for $1 \mathrm{~h}$ with $3 \% \mathrm{BSA}$, the sections were incubated with anti-E-cadherin (1:50) or anti-vimentin (1:100) antibody for $1 \mathrm{~h}$ at room temperature. Immunoreactivity was detected using the biotin-streptavidin-peroxidase complex method and visualized using the 3,3'-diaminobenzidine (DAB; Dako) chromogen. Sections were counterstained with hematoxylin.

A modified semi-quantitative scoring system $(23,24)$ was used to evaluate immunostaining by light microscopy: 0 , no cells stained in any field; 1 , positive staining of $<25 \%$ of cells; 2 , positive staining of $25-50 \%$ of cells; 3 , positive staining of $50-75 \%$ cells; and 4 , positive staining of $>75 \%$ cells. Staining intensity was evaluated using the following scale: 0 , no cell staining; $1+$, mild staining; $2+$, moderate staining; and 3+, strong staining. The total score was generated by adding the scores for the percentage of positive cells and staining intensity together.

Animal experiments. Six-week-old athymic nude mice (nu/nu), obtained from the Chinese Academy of Medical Sciences, were allowed to adapt to the laboratory environment for 1 week. Experiments were then performed as previously described (25). Briefly, SW1736 cells transfected with either scrambled siRNA or EGFR-specific siRNA (EGFR-silenced cells) were injected through the tail vein of female mice. In order to examine the effect of anti-miR-200s in vivo, EGFR-silenced SW1736 cells were also injected into the mice. SW1736 cells $\left(3 \times 10^{6}\right.$ cells/animal) were immobilized in Matrigel $(300 \mathrm{mg} / \mathrm{ml})$ and injected into the mice. Ten mice were included in each treatment group. After 4 weeks, all mice were sacrificed and tumor tissue was removed and processed for immunohistochemistry. All procedures were monitored and approved by the local ethics committee and federal authorities and were conducted in accordance with the guidelines for the welfare of animals in experimental neoplasia.

Statistical analysis. Differences between groups were analyzed by one-way ANOVA using SPSS13.0 software (SPSS, Chicago, IL, USA). P-values $<0.05$ were considered to indicate statistically significant differences. 

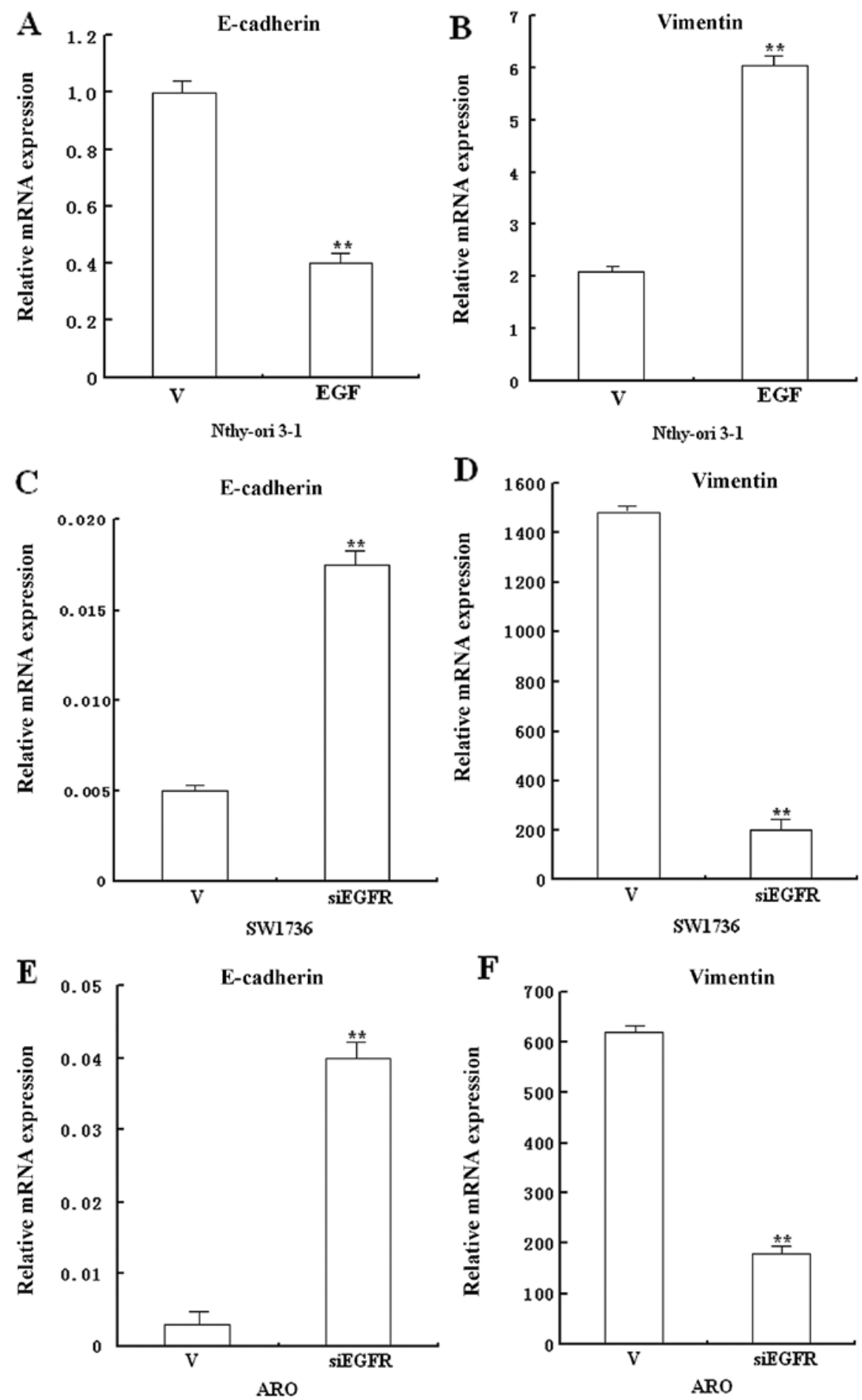

Figure 1. EGF and EGFR siRNA treatments have opposing effects on EMT marker expression. Expression of E-cadherin and vimentin was evaluated by real-time RT-PCR in (A and B) Nthy-ori 3-1, (C and D) SW1736 and (E and F) ARO cells. V, vector control; ${ }^{* *} \mathrm{P}<0.01$, compared to control.

\section{Results}

EGF/EGFR signaling correlates with vimentin, E-cadherin and RhoA expression. The induction of the EGF/EGFR pathway by the EGF treatment of Nthy-ori 3-1 cells resulted in the upregulation of the mesenchymal marker, vimentin, accompanied by the reduced expression of the epithelial marker, E-cadherin, suggesting the induction of an EMT phenotype. By contrast, the downregulation of EGF/EGFR signaling by siRNA-mediated EGFR silencing in SW1736 cells resulted in increased E-cadherin mRNA expression, concomitant with the reduced vimentin expression. To rule out cell line-specific effects, we carried out similar studies using the aggressive thyroid cancer cell line, ARO, and also found that EGFR silencing resulted in a significant increase in E-cadherin mRNA levels and decreased vimentin expression (Fig. 1). We evaluated RhoA expression by western blot analysis, and found that the EGF treatment of Nthy-ori 3-1 cells led to increased RhoA expression, whereas EGFR silencing in SW1736 and ARO cells led to the downregulation of RhoA (Fig. 2). As EGFR silencing 


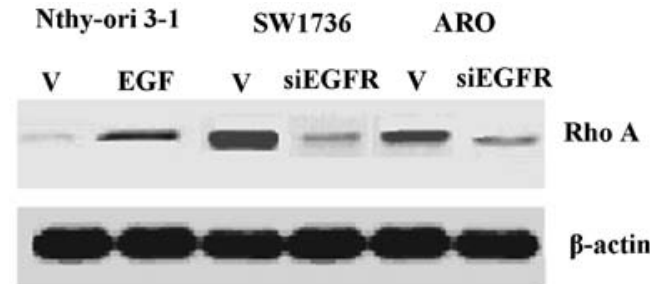

Figure 2. EGF and EGFR siRNA treatments have opposing effects on RhoA expression. RhoA expression in Nthy-ori 3-1, SW1736 and ARO cells was evaluated by western blot analysis. $\beta$-actin was used as the loading control. V, vector control.
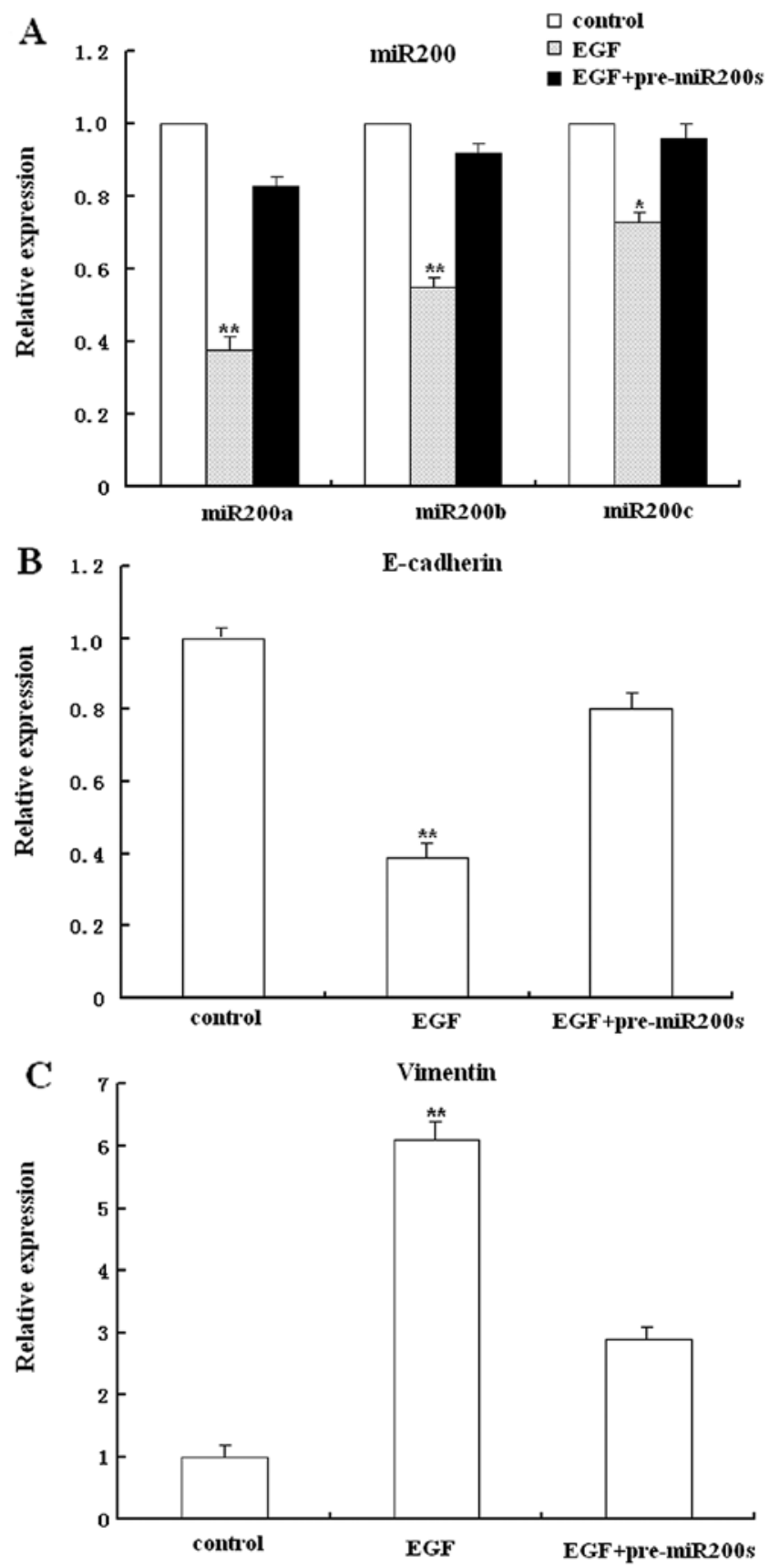

Figure 3. Re-expression of miR-200a/c reverses EGF-induced EMT in Nthy-ori 3-1 cells. (A) Basal expression of miR-200a, miR-200b and miR-200c was evaluated by real-time RT-PCR in Nthy-ori 3-1 cells. (B and C) E-cadherin and vimentin expression in EGF-induced Nthy-ori 3-1 cells treated with pre-miR-200 was evaluated by real-time RT-PCR. " $\mathrm{P}<0.05$ and ${ }^{* *} \mathrm{P}<0.01$, controls vs. EGF-induced cells \pm pre-miRNAs.
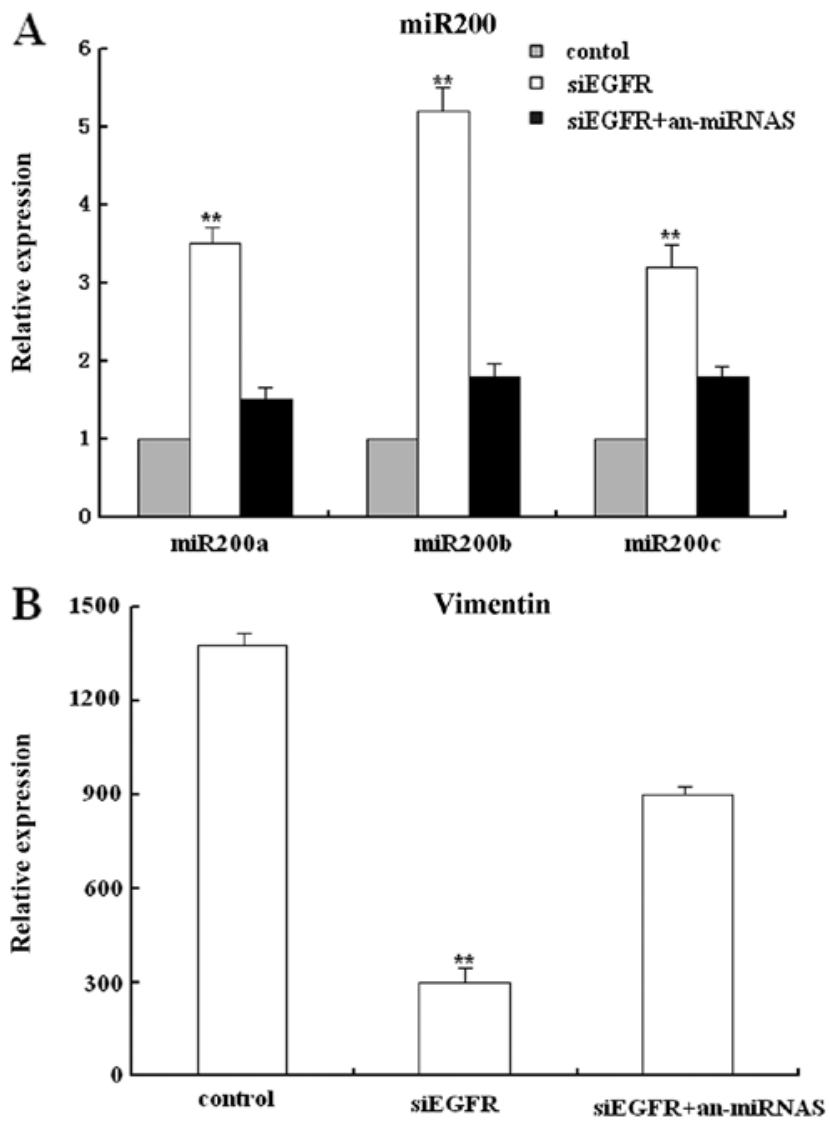

C

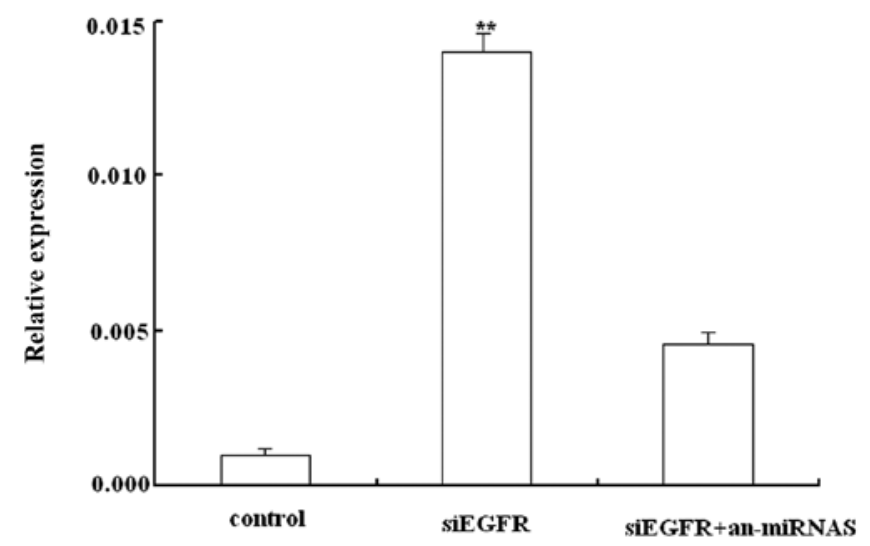

Figure 4. miR-200 suppression blocks EGFR silencing-induced MET in SW1736 cells. (A) Expression of miR-200a, miR-200b and miR-200c was evaluated by real-time RT-PCR in SW1736 cells. (B and C) The effect of suppression of miR-200s in EGFR-silenced SW1736 cells on MET marker expression was evaluated by real-time RT-PCR. ${ }^{* *} \mathrm{P}<0.01$, controls vs. EGFR-silenced cells \pm anti-miRNAs.

in both cell lines had similar effects on EMT markers and Rho/ ROCK signaling, we chose the more experimentally tractable SW1736 cells as a model thyroid cancer cell line for further experiments.

EGF induces downregulation of miR-200s and EMT is reversed by re-expression of miR-200s in Nthy-ori 3-1 cells. In our study, we observed that EGF treatment correlated with reduced miR-200 levels and EMT in Nthy-ori 3-1 cells (Fig. 3A). To assess whether the miR-200 family regulates 


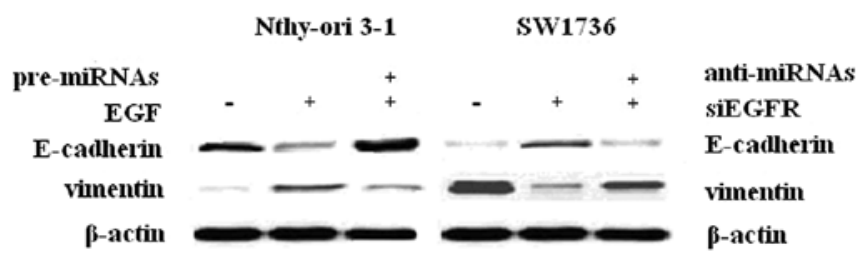

Figure 5. MiR-200s modulate EGF-mediated EMT in thyroid cells Expression of E-cadherin and vimentin EMT markers in Nthy-ori 3-1 cells treated with pre-miR-200 or EGF and SW1736 cells treated with antimiR-200 oligonucleotides or EGFR siRNA. $\beta$-actin was the loading control.

EMT, we transfected EGF-induced Nthy-ori 3-1cells with pre-miR-200a/c to restore miR-200 function. Transfection with pre-miRNAs is a standard technique for inducing the expression of miRNAs (21). The levels of miR-200s in pre$\mathrm{miR}-200 \mathrm{a} / \mathrm{c}$-transfected cells approached the levels of the untreated cells, indicating that miR-200s can be efficiently re-expressed following EGF-mediated downregulation (Fig. 3A). Furthermore, miR-200a/c re-expression resulted in increased E-cadherin expression (Fig. 3B) and decreased vimentin expression (Fig. 3C), thus reversing the mesenchymal phenotype of EGF-treated cells.

EGFR siRNA-mediated MET induction is reversed by miR-200 family inhibition. We found that EGFR silencing leads to increased miR-200 expression in SW1736 cells. Following the suppression of upregulated miR-200s using anti-miR-200 oligonucleotides, miR-200 levels in SW1736 cells were reduced to the levels of the untreated cells, showing an efficient downregulation of miR-200s (Fig. 4A). Moreover, vimentin hypo-expression in EGFR-silenced cells was restored to basal levels following anti-miR-200 treatment (Fig. 4B). Similarly, E-cadherin hyper-expression in EGFR-silenced cells was inhibited by anti-miR-200s treatment (Fig. 4C).
EGF/EGFR regulation of thyroid cell EMT and invasion is reversed by $\mathrm{miR}-200 \mathrm{~s}$. To analyze the miR-200 modulation of EGF-mediated EMT in thyroid cells, western blot analysis was performed to determine the expression of EMT protein markers. The re-expression of miR-200s in EGF-induced Nthy-ori 3-1 cells resulted in re-expression of E-cadherin and suppression of vimentin, whereas the suppression of miR-200s caused the downregulation of E-cadherin and the upregulation of vimentin in EGFR-silenced SW1736 cells (Fig. 5). We investigated whether the EGF/EGFR regulation of the EMT process correlates with thyroid cancer cell invasion using a Matrigel invasion assay. In Nthy-ori 3-1 cells, EGF treatment resulted in increased cell invasion, which was inhibited by the re-expression of the miR-200s. Conversely, EGFR silencing inhibited SW1736 cell invasion, which was restored by the oligonucleotide suppression of miR-200s (Fig. 6).

In vivo evaluation of miR-200 effects on MET in SW1736 xenografts. We analyzed the expression of E-cadherin and vimentin in SW1736 xenografts by immunohistochemistry. Mouse xenografts derived from SW1736 cells transfected with EGFR siRNA targeting showed strong membranous staining of E-cadherin and significantly lower vimentin expression $(\mathrm{P}<0.01$, respectively). By contrast, xenografts derived from SW1736 cells co-transfected with anti-miR-200s and EGFR siRNA had E-cadherin and vimentin expression profiles similar to the xenografts from untransfected cells, indicating that the downregulation of miR-200s reverses the inhibitory effect of EGFR siRNA on EMT. Therefore, miR-200s play a crucial role in EGF/EGFR-mediated EMT in vivo (Fig. 7).

\section{Discussion}

We report that EGF induction in thyroid cancer cells leads to the loss of miR-200 expression and increased Rho/ROCK activity, resulting in increased EMT and subsequent cancer invasion.
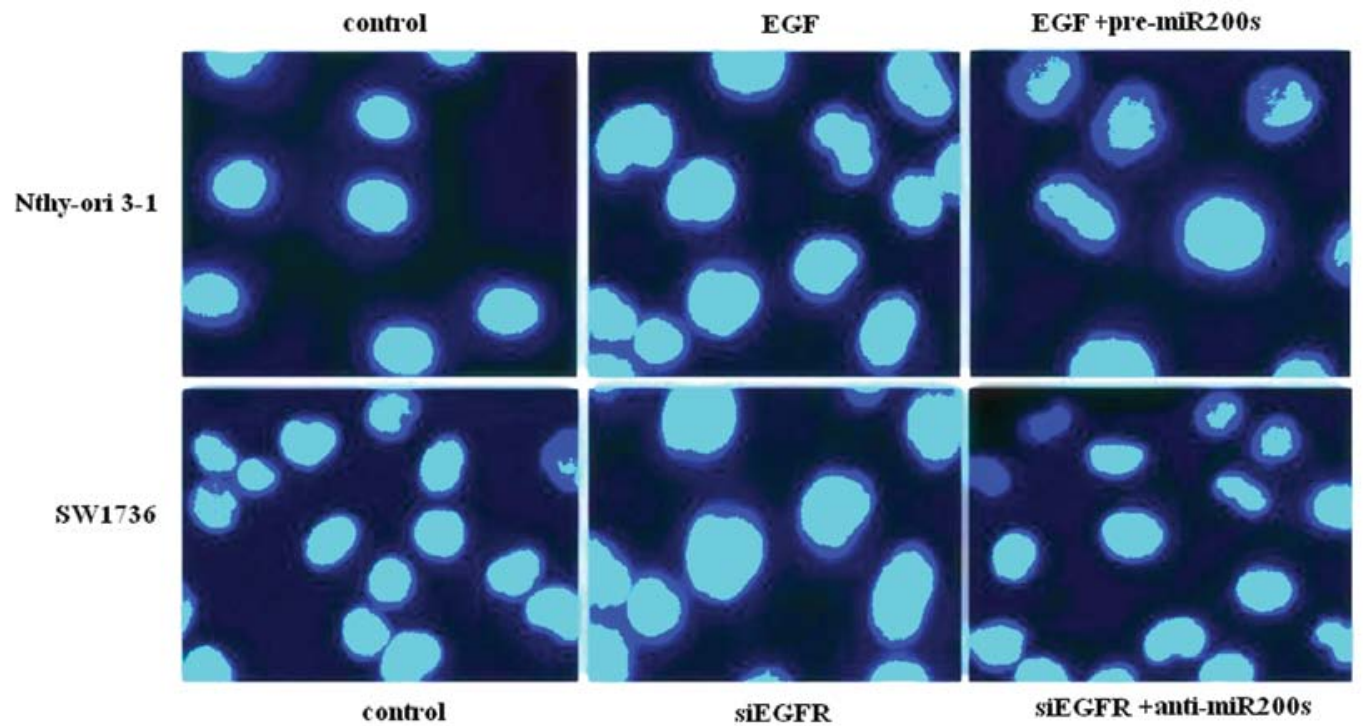

Figure 6. MiR-200s modulate EGF-mediated thyroid cell invasion. Thyroid cell invasion in Nthy-ori 3-1 (upper panel) and SW1736 (lower panel) cells was assayed in Matrigel-coated Transwell chambers. Cells that invaded the Matrigel were stained and photographed using a fluorescence microscope. 
A
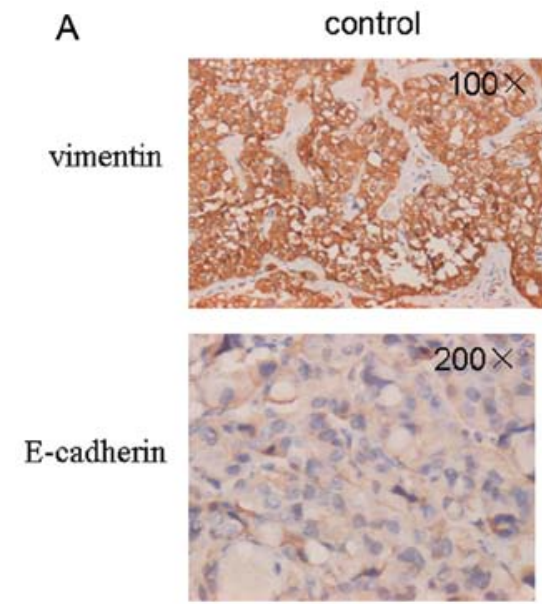

SiEGFR
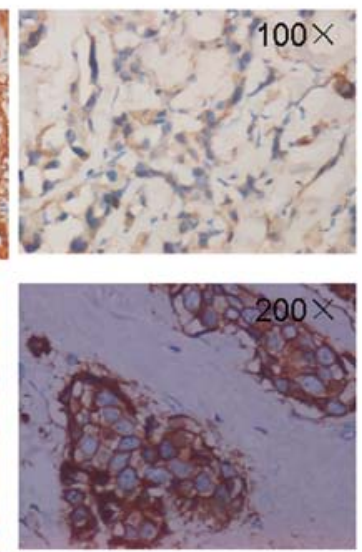

siEGFR+antimiR200s
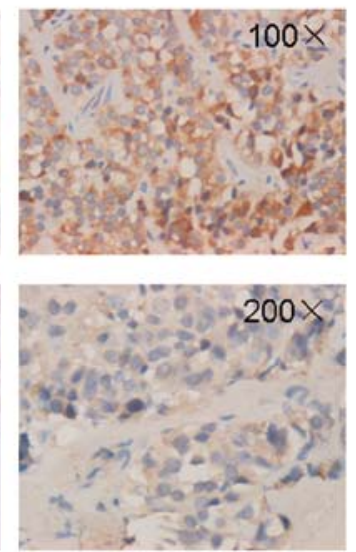

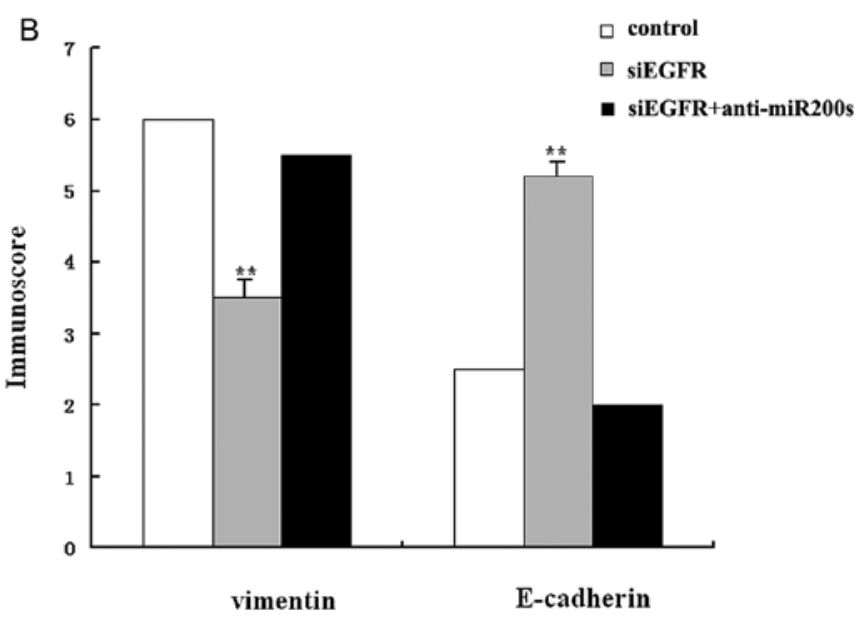

Figure 7. Anti-miR-200 proteins reverse EGFR siRNA-induced EMT in SW1736 cells. (A) Expression of E-cadherin and vimentin in SW1736 xenografts was examined by immunohistochemistry. DAB was used as the chromogen, and the cells were counterstained with hematoxylin. (B) Average semi-quantitative scores of E-cadherin and vimentin stained sections of xenograft tumors. ${ }^{* *} \mathrm{P}<0.01$. Vector control vs. EGFR-silenced cells \pm anti-miRNAs

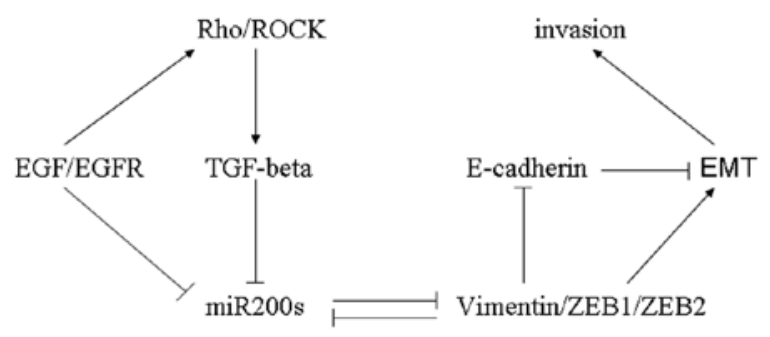

Figure 8. Schematic representation of EGF/EGFR regulation of EMT and thyroid cancer cell invasion. miR-200s play a crucial role in EMT induction via EGF/EGFR. The complex association between miR-200s, Rho/ROCK, TGF- $\beta$, vimentin/ZEB1/ZEB2 and E-cadherin, and their regulation by EGF/EGFR may be important for EMT and aggressive behavior in thyroid cancer cells.

EGF treatment leads to increased vimentin expression in Nthy-ori 3-1 cells, whereas EGFR silencing results in vimentin downregulation in SW1736 cells. Thus, EGF induces EMT in Nthy-ori 3-1 cells and EGFR silencing in SW1736 cells reverses EMT. These observations, combined with reports that the miR-200 family regulates EMT $(19,21)$, led us to hypothesize that the miR-200 family plays a key role in EGF/EGFR-induced EMT in thyroid cells. We found that EGF treatment downregulates miR-200s in Nthy-ori 3-1 cells, whereas EGFR silencing upregulates miR-200s in SW1736 cells. These results show that EGF/EGFR signaling modulates the expression of miR-200s in thyroid cancer cells, thus providing a possible link between EGF/EGFR-mediated EMT and miR-200 family expression.

$\mathrm{Rho} / \mathrm{ROCK}$ is constitutively active in cancer cells and is therefore an attractive therapeutic target $(22,26)$. Rho/ROCK activation is necessary for TGF $\beta$-induced EMT, and inhibition of the Rho effector, ROCK, inhibits TGF $\beta$-induced EMT in vitro and in vivo $(27,28)$. In the current study, we observed increased Rho/ROCK activity in EGF-treated Nthy-ori 3-1 cells, and reduced Rho/ROCK activity in EGFR-silenced SW1736 cells.

Previous studies have indicated that EMT induction is regulated by the miR-200 family (18-21). TGF $\beta$ negatively regulates the expression of the miR-200 family and thereby promotes expression of zinc-finger enhancer binding (ZEB) transcription factors, leading to EMT in vitro (27). In this study, we show that the re-expression of $\mathrm{miR}-200 \mathrm{a} / \mathrm{c}$ by transfection of pre-miR-200 inhibits cell invasion in vitro, thus providing direct evidence in support of the involvement of miR-200s in EGF/EGFR-mediated effects on thyroid cancer cell aggressiveness. Using a reciprocal model in Nthy-ori 3-1 cells, we show that EGF-mediated cell invasion is effectively blocked by the re-expression of the miR-200s. Our in vitro and in vivo results indicate a key regulatory role for miR-200s in the modulation of EMT by EGF/EGFR.

Our current findings, together with existing literature, provide a model for EMT regulation by EGF/EGFR, miR-200s, Rho/ROCK and EMT markers (Fig. 8). We therefore provide evidence for a mechanism linking the miR-200 family with EGF/EGFR signaling, suggesting that miR-200 upregulation may serve as a novel therapeutic strategy for highly invasive thyroid cancer.

\section{Acknowledgements}

The present study was supported by a grant from the Shanghai Municipal Health Bureau Scientific Foundation of China (2010-51). 


\section{References}

1. Riesco-Eizaguirre G and Santisteban P: New insights in thyroid follicular cell biology and its impact in thyroid cancer therapy. Endocr Relat Cancer 14: 957-977, 2007.

2. Patel KN and Shaha AR: Poorly differentiated and anaplastic thyroid cancer. Cancer Control 13: 119-128, 2006.

3. Zhong WB, Liang YC, Wang CY, Chang TC and Lee WS: Lovastatin suppresses invasiveness of anaplastic thyroid cancer cells by inhibiting Rho geranylgeranylation and RhoA/ROCK signaling. Endocr Relat Cancer 12: 615-629, 2005.

4. Larue L and Bellacosa A: Epithelial-mesenchymal transition in development and cancer: role of phosphatidylinositol 30 kinase/ AKT pathways. Oncogene 24: 7443-7454, 2005.

5. Thiery JP, Acloque H, Huang RY and Nieto MA: Epithelialmesenchymal transitions in development and disease. Cell 139: 871-890, 2009.

6. Christiansen JJ and Rajasekaran AK: Reassessing epithelial to mesenchymal transition as a prerequisite for carcinoma invasion and metastasis. Cancer Res 66: 8319-8326, 2006.

7. Bergstrom JD, Westermark B and Heldin NE: Epidermal growth factor receptor signaling activates met in human anaplastic thyroid carcinoma cells. Exp Cell Res 259: 293-299, 2000.

8. Kuan CT, Wikstrand CJ and Bigner DD: EGF mutant receptor vIII as a molecular target in cancer therapy. Endocr Relat Cancer 8: 83-96, 2001

9. Larsen AK, Ouaret D, El Ouadrani K and Petitprez A: Targeting EGFR and $\operatorname{VEGF(R)}$ pathway cross-talk in tumor survival and angiogenesis. Pharmacol Ther 131: 80-90, 2011.

10. Cascone T, Herynk MH, Xu L, Du Z, Kadara H, Nilsson MB, Oborn CJ, Park YY, Erez B, Jacoby JJ, et al: Upregulated stromal EGFR and vascular remodeling in mouse xenograft models of angiogenesis inhibitor-resistant human lung adenocarcinoma. J Clin Invest 121: 1313-1328, 2011.

11. Ferrer-Soler L, Vazquez-Martin A, Brunet J, Menendez JA, Llorens RD and Colomer R: An update of the mechanisms of resistance to EGFR-tyrosine kinase inhibitors in breast cancer: Gefitinib (Iressa)-induced changes in the expression and nucleocytoplasmic trafficking of HER-ligands (Review). Int J Mol Med 20: 3-10, 2007

12. Sanz-Moreno V, Gaggioli C, Yeo M, Albrengues J, Wallberg F, Viros A, Hooper S, Mitter R, Féral CC, Cook M, et al: ROCK and JAK1 signaling cooperate to control actomyosin contractility in tumor cells and stroma. Cancer Cell 20: 229-245, 2011.

13. Zhang Z, Ren JH, Li ZY, Nong L and Wu G: Fasudil inhibits lung carcinoma-conditioned endothelial cell viability and migration. Oncol Rep 27: 1561-1566, 2012.

14. Mavria G, Vercoulen Y, Yeo M, Paterson H, Karasarides M, Marais R, Bird D and Marshall CJ: ERK-MAPK signaling opposes Rho-kinase to promote endothelial cell survival and sprouting during angiogenesis. Cancer Cell 9: 33-44, 2006.

15. Zhong WB, Hsu SP, Ho PY, Liang YC, Chang TC and Lee WS: Lovastatin inhibits proliferation of anaplastic thyroid cancer cells through up-regulation of p27 by interfering with the Rho/ ROCK-mediated pathway. Biochem Pharmacol 82: 1663-1672, 2011.

16. Kidera Y, Tsubaki M, Yamazoe Y, Shoji K, Nakamura H, Ogaki M, Satou T, Itoh T, Isozaki M, Kaneko J, et al: Reduction of lung metastasis, cell invasion, and adhesion in mouse melanoma by statin-induced blockade of the Rho/Rho- associated coiled-coil containing protein kinase pathway. J Exp Clin Cancer Res 29: 127, 2010.
17. Foekens JA, Sieuwerts AM, Smid M, Look MP, de Weerd V, Boersma AW, Klijn JG, Wiemer EA and Martens JW: Four miRNAs associated with aggressiveness of lymph node-negative estrogen receptor-positive human breast cancer. Proc Natl Acad Sci USA 105: 13021-13026, 2008.

18. Ma L, Teruya-Feldstein $\mathrm{J}$ and Weinberg RA: Tumour invasion and metastasis initiated by microRNA-10b in breast cancer. Nature 449: 682-688, 2007.

19. Gregory PA, Bert AG, Paterson EL, Barry SC, Tsykin A, Farshid G, Vadas MA, Khew-Goodall Y and Goodall GJ: The miR-200 family and miR-205 regulate epithelial to mesenchymal transition by targeting ZEB1 and SIP1. Nat Cell Biol 10: 593-601, 2008.

20. Park SM, Gaur AB, Lengyel E and Peter ME: The miR-200 family determines the epithelial phenotype of cancer cells by targeting the E-cadherin repressors ZEB1 and ZEB2. Genes Dev 22: 894-907, 2008.

21. Kong D, Li Y, Wang Z, Banerjee S, Ahmad A, Kim HR and Sarkar FH: miR-200 regulates PDGF-D-mediated epithelialmesenchymal transition, adhesion, and invasion of prostate cancer cells. Stem Cells 27: 1712-1721, 2009.

22. Yin L, Morishige K, Takahashi T, Hashimoto K, Ogata S, Tsutsumi S, Takata K, Ohta T, Kawagoe J, Takahashi K and Kurachi H: Fasudil inhibits vascular endothelial growth factorinduced angiogenesis in vitro and in vivo. Mol Cancer Ther 6: $1517-1525,2007$.

23. Harvey JM, Clark GM, Osborne CK and Allred DC: Estrogen receptor status by immunohistochemistry is superior to the ligand-binding assay for predicting response to adjuvant endocrine therapy in breast cancer. J Clin Oncol 17: 1474-1481, 1999.

24. Umemura S, Itoh J, Itoh H, Serizawa A, Saito Y, Suzuki Y, Tokuda Y, Tajima T and Osamura RY: Immunohistochemical evaluation of hormone receptors in breast cancer: which scoring system is suitable for highly sensitive procedures? Appl Immunohistochem Mol Morphol 12: 8-13, 2004.

25. Wunderlich A, Fischer M, Schlosshauer T, Ramaswamy A, Greene BH, Brendel C, Doll D, Bartsch D and Hoffmann S: Evaluation of Aurora kinase inhibition as a new therapeutic strategy in anaplastic and poorly differentiated follicular thyroid cancer. Cancer Sci 102: 762-768, 2011.

26. Ying H, Biroc SL, Li WW, Alicke B, Xuan JA, Pagila R, Ohashi Y, Okada T, Kamata,Y and Dinter H: The Rho kinase inhibitor fasudil inhibits tumor progression in human and rat tumor models. Mol Cancer Ther 5: 2158-2164, 2006.

27. Cho HJ and Yoo J: Rho activation is required for transforming growth factor-beta-induced epithelial-mesenchymal transition in lens epithelial cells. Cell Biol Int 31: 1225-1230, 2007.

28. Sun GP, Kohno M, Guo P, Nagai Y, Miyata K, Fan YY, Kimura S, Kiyomoto H, Ohmori K, Li DT, Abe Y and Nishiyama A: Involvements of Rho-kinase and TGF-beta pathways in aldosterone-induced renal injury. J Am Soc Nephrol 17: 2193-2201, 2006. 